\title{
Treatments for combined small cell lung cancer patients
}

\author{
Jiaxi He $\mathrm{He}^{1,2,3,4 \#}$, Songhui Xü" Hui Pan ${ }^{1,2,3 \#}$, Shuben $\mathrm{Li}^{1,2,3}$, Jianxing $\mathrm{He}^{1,2,3}$ \\ ${ }^{1}$ Department of Thoracic Surgery, the First Affiliated Hospital of Guangzhou Medical University, Guangzhou, China; ${ }^{2}$ Guangzhou Institute of \\ Respiratory Disease \& China State Key Laboratory of Respiratory Disease, Guangzhou, China; ${ }^{3}$ National Clinical Research Center for Respiratory \\ Disease, Guangzhou, China; ${ }^{4}$ University of Maryland Baltimore, School of Medicine, Baltimore, MD, USA \\ Contributions: (I) Conception and design: J He, S Li; (II) Administrative support: S Li, J He; (III) Provision of study materials or patients: J He, H \\ Pan; (IV) Collection and assembly of data: J He, H Pan, S Xu; (V) Data analysis and interpretation: J He, H Pan, S Xu; (VI) Manuscript writing: All \\ authors; (VII) Final approval of manuscript: All authors. \\ \#These authors contributed equally to this work. \\ Correspondence to: Shuben Li, MD, PhD. Jianxing He, MD, PhD. Department of Thoracic Surgery, the First Affiliated Hospital of Guangzhou \\ Medical University, Guangzhou, China. Email: 13500030280@163.com; hejianxing@hotmail.com.
}

Background: Combined small cell lung cancer (CSCLC) is a subtype of small cell lung cancer (SCLC) which contains both components of SCLC and non-small cell lung cancer (NSCLC). The prognostic outcomes and treatment strategy of it are still unclear. A large-scale retrospective study was performed to investigate proper treatments for CSCLC.

Methods: All cases of CSCLC were identified from the SEER database during the period of 2004-2016. Clinical characteristics, first-line treatments, surgical procedures and survival data including overall survival (OS) and cancer-specific survival (CSS) were analyzed.

Results: A total of 37,639 SCLC patients were identified. CSCLC accounted for $2.1 \%(784 / 37,639)$. The mean age of CSCLC cohort is $67.3 \pm 9.9$ years old. Male and white ethnicity patients were accounted for larger proportions (55.7\% and 80.4\%). The oncological characteristics of CSCLC were consistent with SCLC that most of patients were diagnosed as higher grade and advanced stages. The prognosis of CSCLC was better than SCLC but worse than NSCLC in IA-IIIA stages. No difference was observed in IIIBIV. Surgery was beneficial in IA-IB stage CSCLC. Adjuvant chemotherapy seemed to have few effects on early stage patients. Trimodality treatment could significantly improve OS in IIA-IIIA CSCLC patients. Chemotherapy-based treatment is predominant choice in advanced stage patients.

Conclusions: CSCLC is a rare and special subtype of SCLC. It has better survival outcome than nonCSCLC in early stage. Surgical treatment is crucial in early stage of CSCLC. Prognostic improvement might be achieved from trimodality treatment in stage IIA-IIIA. Chemotherapy-based treatments should be considered in advanced stage. The effect of surgical treatments in advanced stage patients should be further investigated.

Keywords: Combined small cell lung cancer (CSCLC); SEER; surgery; prognosis; treatment

Submitted Mar 16, 2020. Accepted for publication Aug 03, 2020.

doi: $10.21037 /$ tlcr-20-437

View this article at: http://dx.doi.org/10.21037/tlcr-20-437

\section{Introduction}

According to the World Health Organization (WHO) Histology Criteria 2015, combined small cell lung cancer (CSCLC) is categorized as a subtype of small cell lung cancer (SCLC) $(1,2)$. It is diagnosed via pathological specimen containing SCLC component and any other malignant components including adenocarcinoma (AD), squamous cell carcinoma (SQ) and large cell carcinoma. Some rare histological types, such as giant cell carcinoma, spindle cell carcinoma and sarcomatoid malignancy, also can be seen in CSCLC. It can be diagnosed regardless of cell amounts when SCLC coexisting with AD, SQ or 
sarcomatoid carcinoma. In terms of large cell carcinoma, at least $10 \%$ of large cell carcinoma component should be observed to make the diagnosis $(2,3)$. In the previous studies, more than two components of NSCLC were reported in CSCLC (3).

The previous studies indicated that CSCLC accounted for $5-14 \%$ of SCLC (4-8). The actual incidence may be higher than the previous report because most of CSCLC cases were diagnosed from surgical specimens. The biopsy specimens from CT-guided thoracentesis, bronchoscopy and EBUS are difficult to conclude proper diagnosis. Some retrospective studies showed that CSCLC shared similar characteristics and epidemiological features with SCLC. Besides male and smoking patient predominance, most cases of CSCLC belonged to advanced stages when they were firstly diagnosed. Zhang et al. reported almost $90 \%$ of CSCLC were diagnosed as stage III and IV in their cohort (9).

Referring to the treatments for SCLC, platinum-based etoposide chemotherapy with/without radiotherapy is recommended for SCLC patients in advanced stages by the National Comprehensive Cancer Network (NCCN) guidelines. Surgical treatment is always a controversial topic in early stage of SCLC. A few previous studies reported no prognostic difference was observed between surgical and non-surgical treatment SCLC patients. Some of them even reported worse outcomes in surgical groups (10-13). Most of these studies included stage II and III patients or ignored the effect of adjuvant chemotherapy. Yang et al. reported that pT1-2N0M0 SCLC patients could be beneficial from adjuvant chemotherapy with/without cranial irradiation (14). Moon and his colleagues reported that surgery with chemoradiation provided better cancer-specific survival (CSS) in T1-2N0-1M0 SCLC while CSCLC patients might benefit from multimodality (8). The optimal treatments for CSCLC in each stage are not fully verified.

We therefore sought to demonstrate the clinical characteristics and prognosis of CSCLC based on a largesize sample. Moreover, we also try to explore the optimal treatment for each stage CSCLC for the sake of better prognosis. We present the following article in accordance with the STROBE reporting checklist (available at http:// dx.doi.org/10.21037/tlcr-20-437).

\section{Methods}

\section{Data source}

The primary cohort of this retrospective study was identified from the Surveillance, Epidemiology and End Result cancer database (SEER), which is maintained and managed by National Cancer Institute (NCI) and is representing approximately $28 \%$ population of United State (15). To achieve maximum inclusion, the database of the most registered centers was chosen in SEER for data extraction. All procedures performed in this study were in accordance with the Declaration of Helsinki (as revised in 2013). Since the study is based on a publicly available database, the approval by the Ethics Committee of the Guangzhou Medical University First Affiliated Hospital and the informed consent had been waived.

\section{Inclusive and exclusive criteria}

Patients diagnosed as primary malignancies at the site of main bronchus and lung (SEER primary site code = C340-C349) from 2004 to 2016 were identified. The histology codes of SCLC were 8041-8045, while CSCLC was 8045 . The data of clinical, oncological characteristics and survival outcomes including overall survival (OS) and cancer-specific survival (CSS) was identified and recorded. Survival data of NSCLC patients in the same period was also extracted. Patients with missing data of stage, tumor size and treatments etc. were subsequently excluded.

\section{Statistical analysis}

The primary outcome was OS. The Kaplan-Meier method and log-rank test were used to demonstrate survival status of the cohort and assess the prognostic differences between various treatments. Univariate and multivariate analysis was performed incorporating gender, age, surgery, stage, adjuvant chemotherapy and radiation. The factors which were considered as collinear would not be analyzed in the same Cox model. CSS was also analyzed in order to minimize the influences from other causes of death.

The Kaplan-Meier and Cox regression were performed using SPSS 25 (IBM) and Prism 8 (Graphpad). Hazard ratio (HR) and the $95 \%$ confident interval $(95 \% \mathrm{CI})$ were reported. The statistical difference was considered as significant when $\mathrm{P}<0.05$. All tests were two-sided.

\section{Results}

\section{Clinical features of primary cohort}

A total of 37,639 SCLC patients were identified in the primary cohort. After applying the exclusive criteria, 


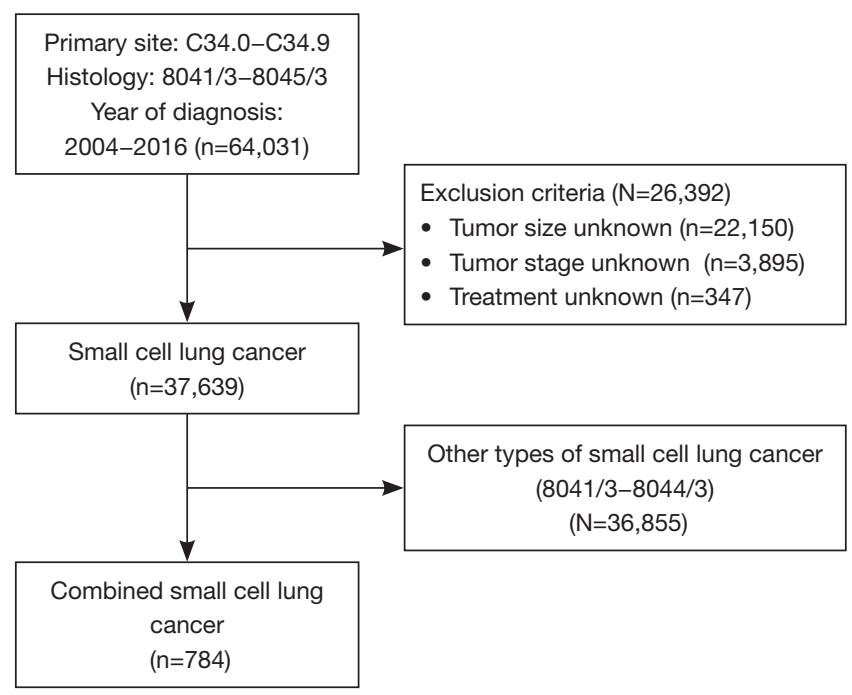

Figure 1 The selection process of the primary cohort.

patients diagnosed as CSCLC were finally included in the primary cohort $(\mathrm{N}=784)$. The selection process was shown in Figure 1. CSCLC accounted for $2.1 \%(784 / 37,639)$. The mean age of CSCLC cohort is $67.3 \pm 9.9$ years old, which is similar to other SCLC $(66.8 \pm 10.1)$. Male patients accounted for a slightly larger proportion $(437 / 784,55.7 \%)$. Most of the patients were white ethnicity $(630 / 784,80.4 \%)$. Referring to the tumor characteristics, the mean tumor size was $45.7 \pm 29.6 \mathrm{~mm}$. Upper lobe was the predominant site of CSCLC which was in consistent with SCLC. Most of the patients were diagnosed as poor differentiation and undifferentiated $(388,49.5 \%)$, though grades of $45 \%$ patients were unknown. A large proportion of CSCLC were diagnosed as advanced stages (IIIB $11.9 \%$, IV 46.6\%). A total of 240 patients underwent surgical treatments, while 361 patients received radiation and 515 patients received chemotherapy (Table 1).

\section{Survival outcomes of CSCLC in stages}

Besides CSCLC, the survival data of other SCLC and NSCLC in the same stages was also analyzed. In stage IA-IB patients, NSCLC has superior survival outcomes both in OS compared to SCLC. The median survival of CSCLC was 18 months, which was better than 13 months of other SCLC (P1=0.003). However, it was not as good as NSCLC, whose median survival was 63 months
$(\mathrm{P} 2<0.0001)$ (Figure 2A). No prognostic difference was observed between CSCLC and other SCLC in stage IIAIIIA patients $(\mathrm{P} 1=0.791)$. The median survival of OS in two groups were identical (11 months). NSCLC patients in the same stages had better OS than CSCLC patients, which had 21 months median survival $(\mathrm{P} 2<0.0001)$ (Figure $2 B)$. In the advanced stages of IIIB and IV, no prognostic superiority could be observed among three groups (CSCLC vs. SCLC P1=0.644; CSCLC vs. NSCLC P2=0.837). The median survival of CSCLC was the same as other SCLC which was only 9 months. Meanwhile, NSCLC patients had 8 months of median survival (Figure 2C). In terms of CSS, the comparative results were similar to OS showing that NSCLC had best prognosis in stage IA-IIIA, while CSCLC had more ideal prognosis than SCLC in early stages. No difference was observed among these groups in advanced stages patients (Figure S1).

The characteristics and treatments of CSCLC patients were included in the multivariate analysis. It showed that older ages, poorer differentiation and higher stages had worse prognosis. Besides, surgical treatment and chemotherapy could improve survival outcomes. Other characteristics including race, sex, primary site and laterality seemed to have few impacts on the prognosis of CSCLC patients. Radiation therapy might be beneficial to survival, but not statistically significant (Figure 3).

\section{Survival outcomes of CSCLC in different treatments}

Majority of early stage patients received surgeries, while patients with advanced stage diseases tended to received non-surgical treatments. The number of patients received chemotherapy or radiation was increasing from early stages to advanced stages. In terms of treatment groups, surgeries with or without adjuvant chemotherapy are predominant in stage IA-IB patients. Chemotherapy with or without radiation and trimodality are mostly given to stage IIIA-IV patients (Table 2).

More specifically, surgeries with or without adjuvant chemotherapy and trimodality brought similar median survival to stage IA-IB CSCLC patients, which were 58, 57 and 53 months, respectively. Patients received chemoradiation had worse OS whose median survival was 23 months $(\mathrm{P}=0.034)$ (Figure $4 A)$. Referring to surgical approaches, we noticed that patients received sublobectomy 
Table 1 The clinical and pathological characteristics of SCLC lung cancer patients

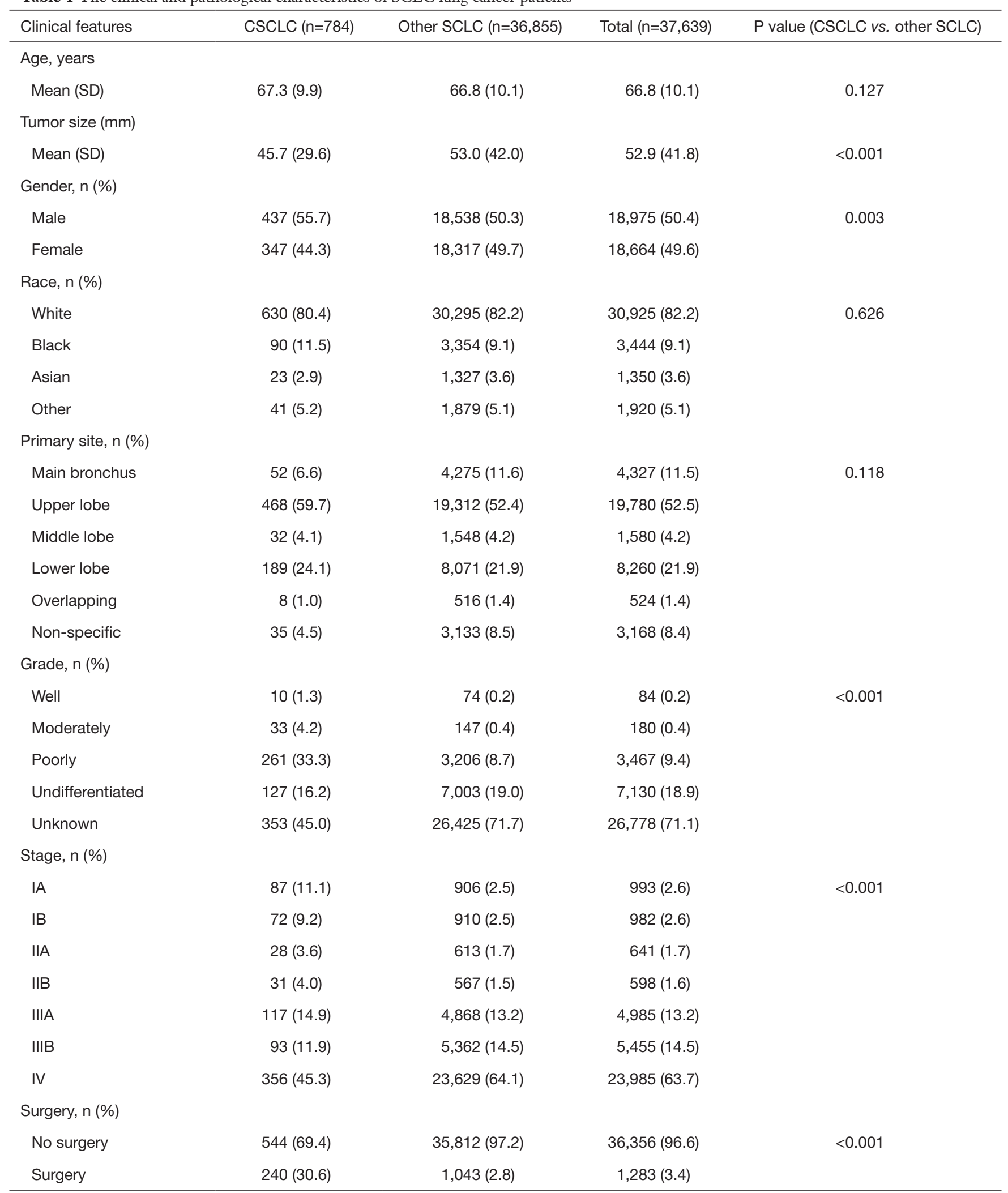

Table 1 (continued) 
Table 1 (continued)

\begin{tabular}{|c|c|c|c|c|}
\hline Clinical features & CSCLC $(n=784)$ & Other SCLC $(n=36,855)$ & Total $(n=37,639)$ & $P$ value (CSCLC vs. other SCLC) \\
\hline No radiation & $423(54.0)$ & $18,570(50.4)$ & $18,993(50.5)$ & \multirow[t]{2}{*}{0.048} \\
\hline Radiation & $361(46.0)$ & $18,285(49.6)$ & $18,646(49.5)$ & \\
\hline \multicolumn{5}{|c|}{ Chemotherapy, n (\%) } \\
\hline Yes & $515(66.7)$ & $26,682(72.4)$ & $27,197(72.3)$ & $<0.001$ \\
\hline
\end{tabular}

CSCLC, combined small cell lung cancer; SCLC, small cell lung cancer.

and lobectomy had similar survival outcomes, which were 58 and 57 months of median survival $(\mathrm{P}=0.6979)$ (Figure S2).

In the stage IIA to IIIA patients, either surgery or chemotherapy alone only had 9 and 8 months median survival. While those received chemoradiation therapy and adjuvant chemotherapy had better survival outcomes than surgery or chemotherapy alone (median survival: 15 and 21 months, $\mathrm{P}=0.9144)$. Patients received trimodality therapy could achieve 30 months of median survival, which was significantly better than other treatments $(\mathrm{P}=0.03)$ (Figure 4B).

In the advanced stages CSCLC patients including IIIB and IV, trimodality therapy could improve their survival outcomes compared to other therapies $(\mathrm{P}<0.001)$. However, no significantly different prognosis was shown among chemotherapy, adjuvant chemotherapy and chemoradiation therapy, which provided median survival of 10 and 12 months. A total of 42 patients received radiation alone had the worst prognosis, whose median survival was only 2 months (Figure 4C).

\section{Discussion}

CSCLC is a rare histological type of SCLC, which is characterized by coexistence of SCLC and other malignancy components in the one tumor. The incidence of CSCLC in previous reports varied from 1-14\%. Fushimi et al. reported the incidence of CSCLC from autopsy was $14.3 \%$, which was significantly higher than $8.6 \%$ from biopsy or other cytological methods (7). Most of the previous studies, which had reported the incidence of CSCLC, were concluded from surgical samples. Such variation may attribute to the different amount of tissue from surgical sample and biopsy specimen. Since chemotherapy was the predominant therapy in SCLC, some cases of CSCLC would be assigned to chemotherapy rather than surgery once their biopsy indicated SCLC. As a result, they might be misdiagnosed as non-CSCLC because of insufficient tissue, which also led to a relatively low incidence. Moon and his colleagues used the same database to study early stage of SCLC from 1988 to 2014. They reported the CSCLC cases accounted for $6.4 \%$ of SCLC in their cohort (8). However, the proportion of CSCLC was just $2.1 \%$ in our cohort. A large amount of advanced stage SCLC cases were included might lead to such difference.

Are the clinical features and prognosis of CSCLC similar to SCLC? Is there anything in common between CSCLC and NSCLC? These questions are the main focuses of researchers since CSCLC has both components. The current study showed that male and old patients account for larger proportion. Besides, the predominant primary site of CSCLC was upper lobe. In terms of differentiation and stage, most of CSCLC patients were diagnosed as poorly differentiation and advanced stages, respectively. These features were identical to SCLC which had also been reported in previous researches. However, the prognosis of CSCLC was better than other non-CSCLC in IA-IIIA stages, though both of them were inferior to NSCLC in the same stages. These results indicated that SCLC component was a negative prognostic factor. Some researchers tried to investigate the correlation between prognosis and proportion of SCLC component in CSCLC. But it still remains unclear. Nevertheless, no difference of OS was observed in stage IIIB and IV among these groups.

Surgical treatment has been proved to be beneficial not 
A IA-IB CSCLC overall survivall

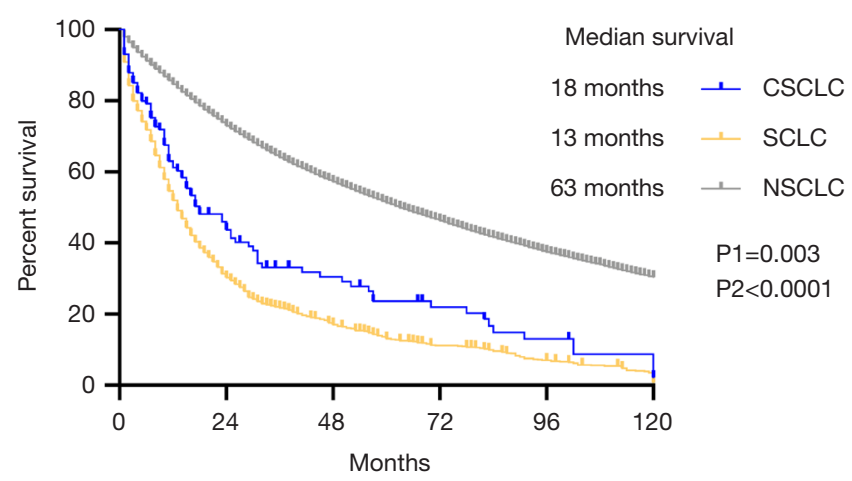

B

IIA-IIIA CSCLC overall suirvival

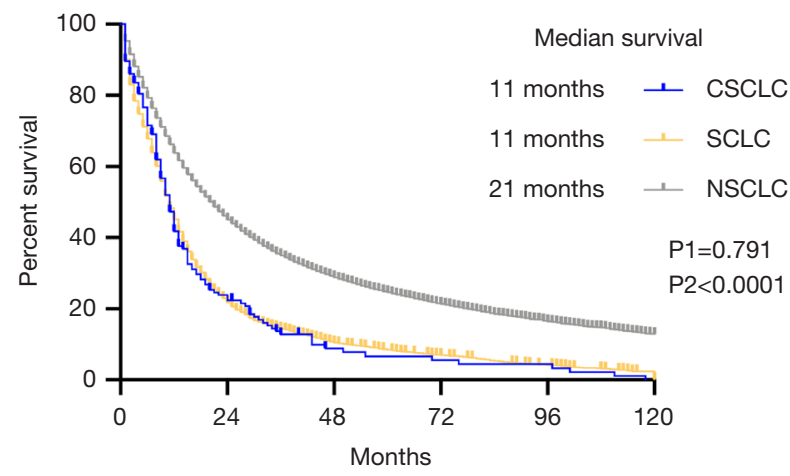

C

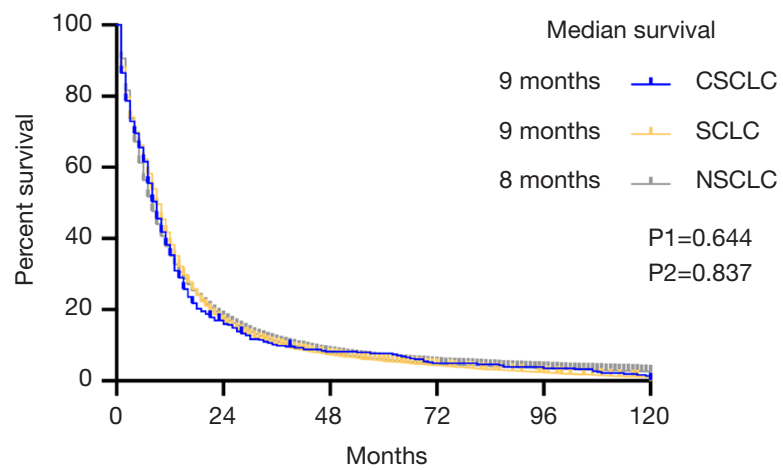

Figure 2 The survival outcomes of CSCLC, other SCLC and NSCLC patients. (A) The overall survival of stage IA-IB patients; (B) the overall survival of stage IIA-IIIA patients; (C) the overall survival of stage IIIB-IV patients. CSCLC, combined small cell lung cancer; SCLC, small cell lung cancer; NSCLC, non-small cell lung cancer. only CSCLC but also SCLC patients $(5,8,14,16)$. Yang et al. reported the pT1-2N0M0 SCLC patients had improved OS from $\mathrm{R} 0$ resection. If adjuvant chemotherapy with/without cranial radiation was administered, the OS would be even better (14). Another Japanese group reported a similar result that postoperative SCLC patients had relatively improved OS which was $52.6 \%$ and $68 \%$ in stage I and II, respectively (17). Babakoohi and his colleagues stated that CSCLC patients were more likely to receive surgical treatment in their cohort. Standard SCLC chemotherapy medications were given to these patients. They noticed that CSCLC patients had better OS than SCLC patients, which was consistent with our findings (5). Men et al. reported that CSCLC patients with surgical treatment had higher 5-year survival rate than nonsurgical treatment patients (16). Moon et al. also used SEER database to investigate treatment on early stage SCLC. They reported that CSCLC patients might benefit from multimodal treatments including surgery than SCLC patients (8). We observed the similar results in IA-IB CSCLC patients as previous studies, which indicated the importance of surgical treatment in early stage of CSCLC. But no superiority of adjuvant chemotherapy was shown in the same patient group. In stage IIA-IIIA patients, we noticed that surgery with chemotherapy or chemoradiation could significantly improve survival. Besides, trimodality treatment brought 30 months median survival to these patients, which indicated that such treatment could be considered if it was appropriate after thorough clinical examinations and evaluations. Referring to distant advanced CSCLC patients, chemotherapy with or without radiation were major options though they had similar prognosis. Although trimodality treatment could significantly improve survival outcomes, it was inappropriate to conclude that surgical treatment should be given to these patients. Given the fact that surgical treatments were not routinely performed in advanced stage patients, some of these patients were probably accidentally diagnosed as higher stages during or after surgeries. The benefits of surgical treatments might be from the debulking effect of tumors or salvage surgeries. Some studies indicated that surgical treatments were beneficial to oligometastatic NSCLC patients and malignant pleural effusion $(18,19)$. No information was provided to determine whether these patients were oligometastatic CSCLC or not. A further study of advanced stage patients is warranted.

Limitations have to admitted in the current study. Frist, no information of comorbidity or smoking history was provided in SEER database. Although CSS was used 


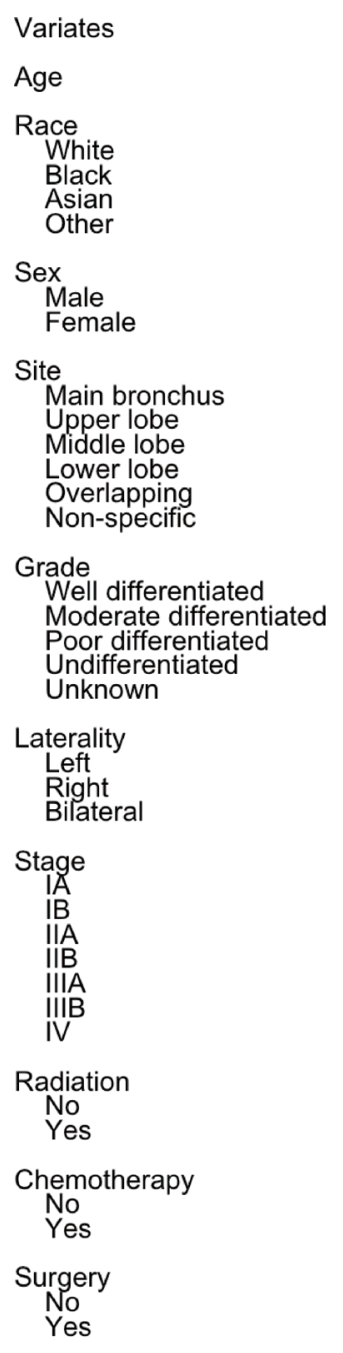

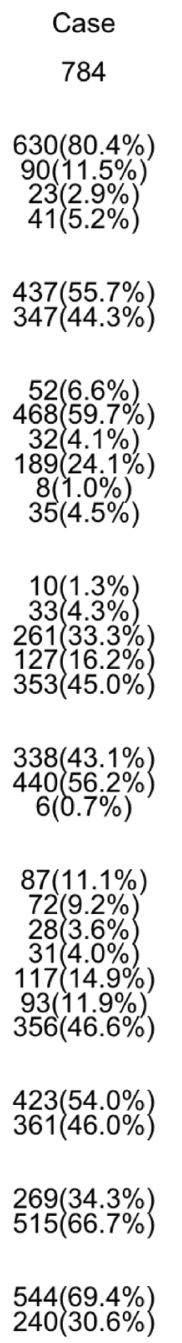

$\mathrm{HR}(95 \% \mathrm{Cl})$

p-value

$1.012(1.004-1.021)$

0.005

Reference

0.768(0.594-0.991

$0.781(0.482-1.264$

$0.917(0.652-1.290)$

0.042
0.314
0.62

0.042
0.314
0.62

Reference

0.890(0.760-1.044)

0.152

Reference

0.772(0.569-1.047

1.114(0.691-1.796

$1.152(0.830-1.600)$

$1.311(0.605-2.841)$

$1.285(0.816-2.023)$

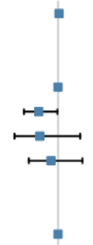

Reference

$2.007(0.867-4.648)$

1.965 (0.914-4.224)

$2.245(1.030-4.895)$

2.099(0.976-4.512)

$m$

0.096

0.659

0.397

0.492

0.279

Reference

$1.022(0.871-1.200)$

$0.989(0.398-2.456)$

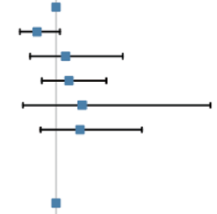

0.104

0.084

0.042

0.058

0.791 1.507(1.005-2.261)

2.563 1.467-4.477

2.114 1 245-3.590

3.352 2.297-4.812

$2.778(1.875-4.116$

$2.778(1.875-4.116)$
$5.869(4.131-8.338)$

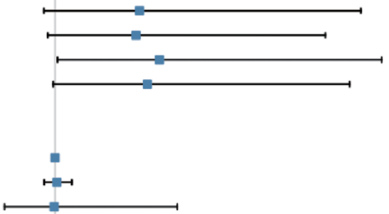

0.981

0.048

0.001

0.006

0.001

0.001

0.001

$0.908(0.766-1.076) \quad 0.264$

0.001

Reference
$0.452(0.374-0.546)$

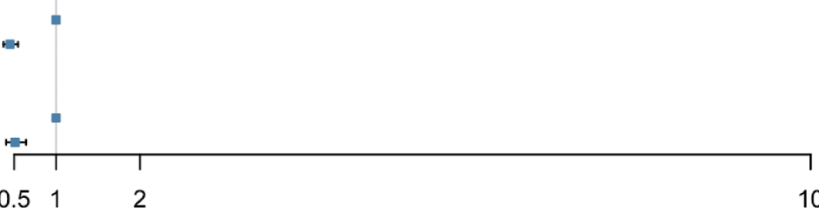

Reference $0.511(0.405-0.644)$

0.001

Figure 3 The multivariate survival analysis of characteristics in CSCLC patients. HR, hazard ratio.

to minimize the interference from other cause of death, comorbidity and adverse events were critical evidence in choosing proper treatment regimens. Smoking history is considered as a crucial risk factor in SCLC. However, very few adenocarcinoma patients have smoking histories. Therefore, such preference is also important in the study to depict characteristics of CSCLC. Second, neither regimens nor sequence of chemotherapy was reported. According to NCCN guidelines, platinum-based etoposide regimen is the first choice for SCLC. Meanwhile, recommendations in NSCLC are completely different. It is generally believed that EP would be usually given to CSCLC patients since the SCLC is more invasive than NSCLC. Given the fact that the ratio of SCLC and NSCLC contents in CSCLC varied, further studies are warranted to determine proper chemotherapy regimen related to the content ratio. Furthermore, the effect of neo-adjuvant chemotherapy for CSCLC was not capable to elucidate owing to lacking of chemotherapy sequence. Mediastinal and distant lymph node involvement was the main reason which hindered surgeons to perform surgery in stage IIIA-IIIB. Regression of involved lymph node and primary tumor after neoadjuvant chemotherapy might provide a good opportunity to patients to receive radical operations. No EGFR mutation 
Table 2 The numbers of CSCLC patients in each treatment groups in different stages

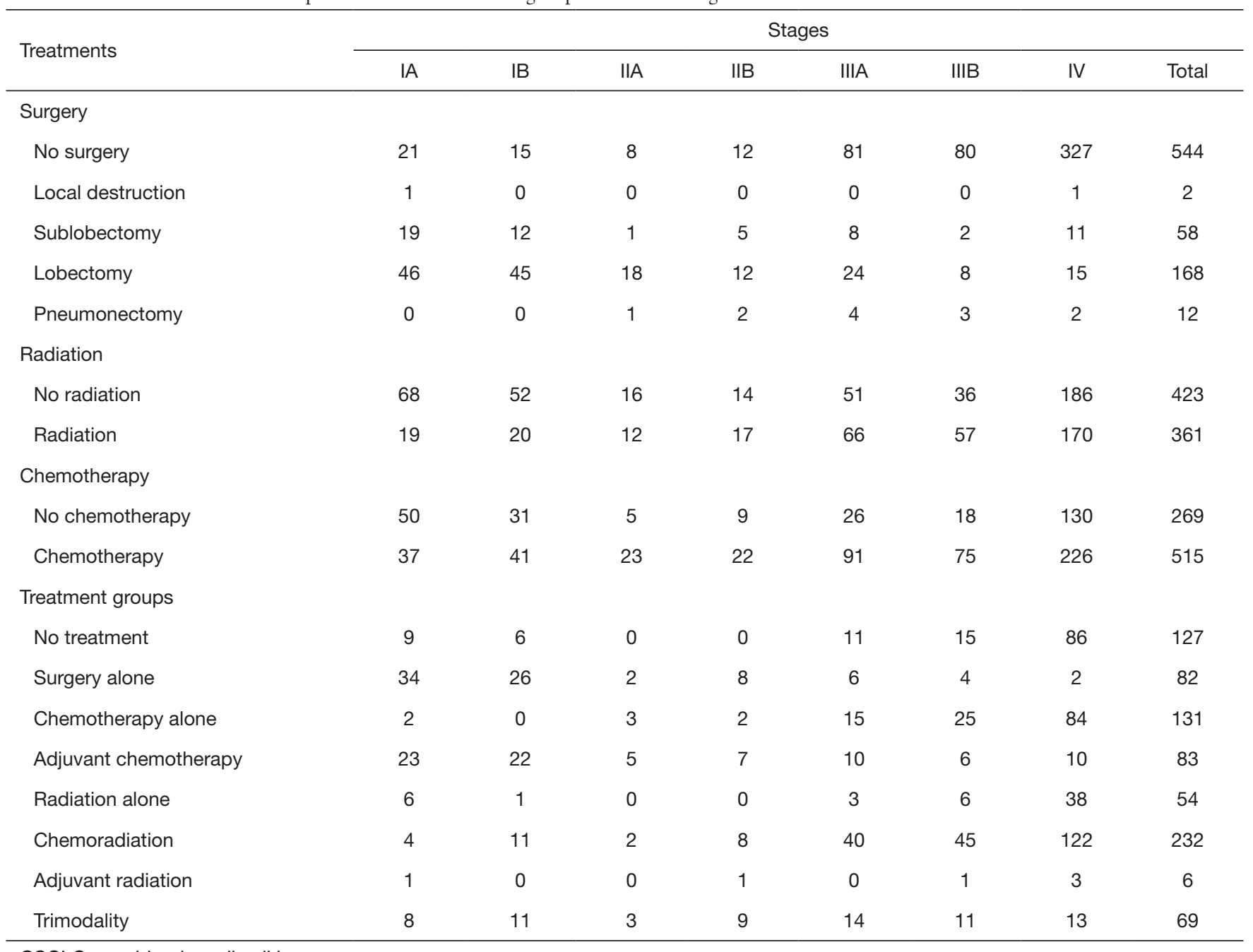

CSCLC, combined small cell lung cancer.

status was provided in the database. Transformation from NSCLC to SCLC has been proved as one of EGFR-TKI resistant mechanism (20,21). Does primary CSCLC also have such resistance? Is the proportion of adenocarcinoma associated with effect of EGFR-TKI? Further studies are warranted to elucidate these questions. Finally, the current study is a retrospective which would generate bias from case selection, statistical analysis and conclusion. Considering CSCLC is a rare disease with low incidence, moderate selection criteria are applied to maximize data acquisition.

\section{Conclusions}

In summary, CSCLC is a rare and special subtype of SCLC. The combined histological characteristics makes CSCLC have a unique clinical feature and treatment strategy. On the other, combined components also make the treatment for CSCLC become complicated. Based on a large-scale database, we found that surgical treatment is crucial in stage IA-IB patients. Prognostic improvement might be achieved from the combination of surgery, chemotherapy and radiation therapy in stage IIA-IIIA. In the advanced 


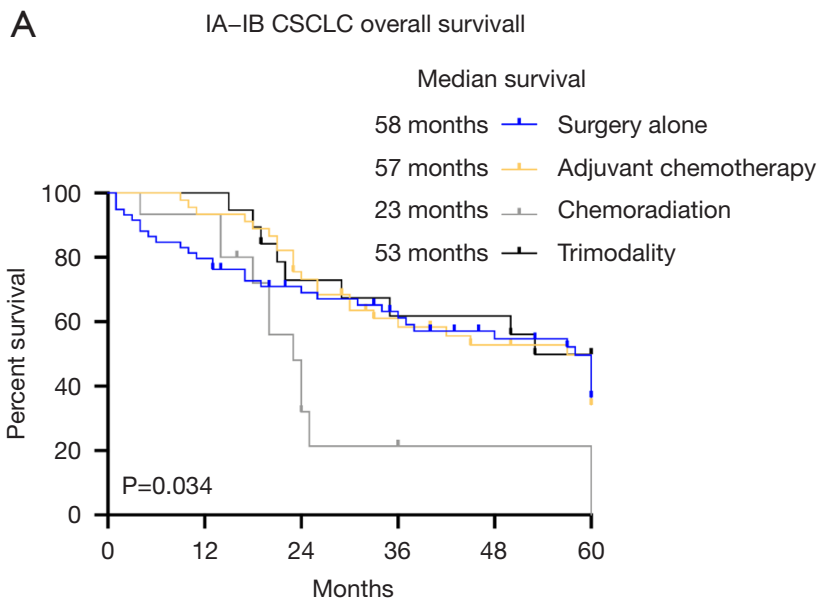

B

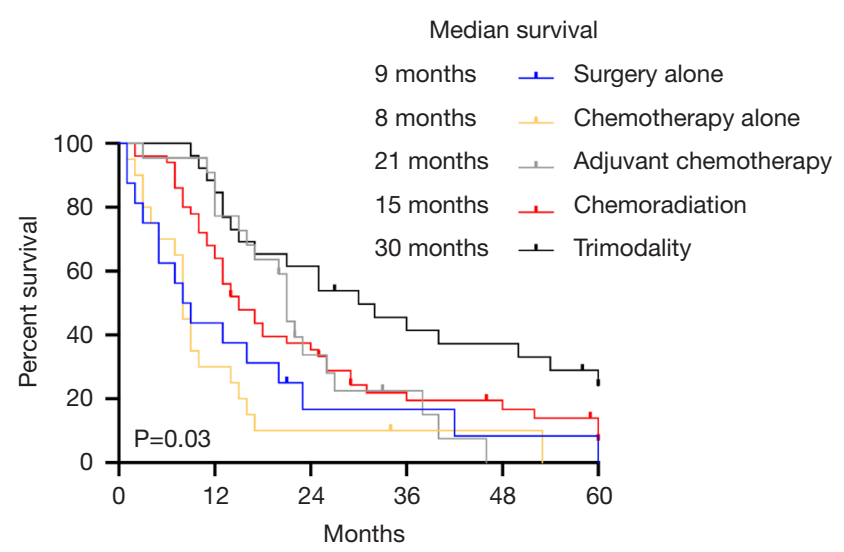

C IIIB-IV CSCLC overalll survival

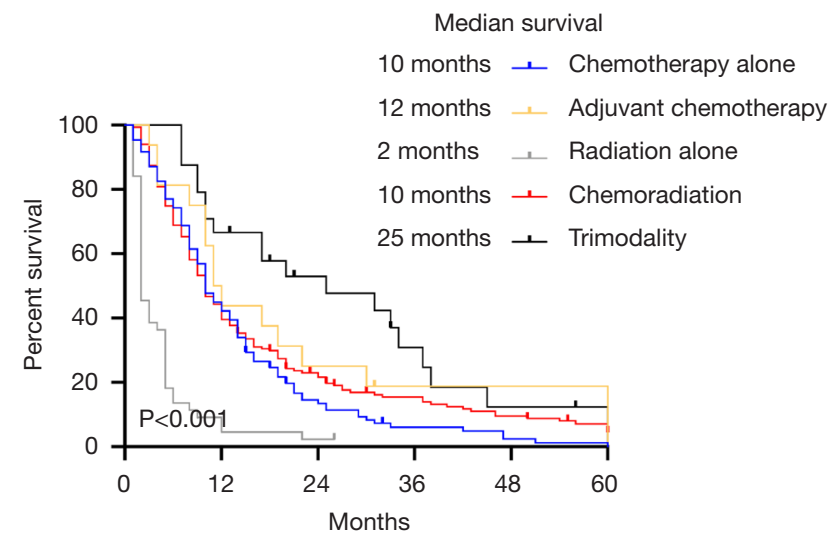

Figure 4 The overall survival of CSCLC received different treatments. (A) The overall survival of Stage IA-IB CSCLC patients; (B) the overall survival of Stage IIA-IIIA CSCLC patients; (C) the overall survival of Stage IIIB-IV CSCLC patients. stage patients, chemotherapy-based treatments should be considered. The effect of debulking surgery should be further investigated.

\section{Acknowledgments}

Funding: None.

\section{Footnote}

Reporting Checklist: The authors have completed the STROBE reporting checklist. Available at http://dx.doi. org/10.21037/tlcr-20-437

Peer Review File: Available at http://dx.doi.org/10.21037/ tlcr-20-437

Conflicts of Interest: All authors have completed the ICMJE uniform disclosure form (available at http://dx.doi. org/10.21037/tlcr-20-437). The authors have no conflicts of interest to declare.

Ethical Statement: The authors are accountable for all aspects of the work in ensuring that questions related to the accuracy or integrity of any part of the work are appropriately investigated and resolved. All procedures performed in this study were in accordance with the Declaration of Helsinki (as revised in 2013). Since the study is based on a publicly available database, the approval by the Ethics Committee of the Guangzhou Medical University First Affiliated Hospital and the informed consent had been waived.

Open Access Statement: This is an Open Access article distributed in accordance with the Creative Commons Attribution-NonCommercial-NoDerivs 4.0 International License (CC BY-NC-ND 4.0), which permits the noncommercial replication and distribution of the article with the strict proviso that no changes or edits are made and the original work is properly cited (including links to both the formal publication through the relevant DOI and the license). See: https://creativecommons.org/licenses/by-nc-nd/4.0/.

\section{References}

1. Mengoli MC, Longo FR, Fraggetta F, et al. The 2015 World Health Organization Classification of lung tumors: new entities since the 2004 Classification. Pathologica 
2018;110:39-67.

2. Travis WD, Brambilla E, Nicholson AG, et al. The 2015 World Health Organization Classification of Lung Tumors: Impact of Genetic, Clinical and Radiologic Advances Since the 2004 Classification. J Thorac Oncol 2015;10:1243-60.

3. Nicholson SA, Beasley MB, Brambilla E, et al. Small cell lung carcinoma (SCLC): a clinicopathologic study of 100 cases with surgical specimens. Am J Surg Pathol 2002;26:1184-97.

4. Govindan R, Page N, Morgensztern D, et al. Changing epidemiology of small-cell lung cancer in the United States over the last 30 years: analysis of the surveillance, epidemiologic, and end results database. J Clin Oncol 2006;24:4539-44.

5. Babakoohi S, Fu P, Yang M, et al. Combined SCLC clinical and pathologic characteristics. Clin Lung Cancer 2013;14:113-9.

6. Tatematsu A, Shimizu J, Murakami Y, et al. Epidermal growth factor receptor mutations in small cell lung cancer. Clin Cancer Res 2008;14:6092-6.

7. Fushimi H, Kikui M, Morino H, et al. Histologic changes in small cell lung carcinoma after treatment. Cancer 1996;77:278-83.

8. Verma V, Choi JI, Simone CB 2nd. Proton therapy for small cell lung cancer. Transl Lung Cancer Res 2018;7:134-40.

9. Zhang C, Yang H, Zhao H, et al. Clinical outcomes of surgically resected combined small cell lung cancer: a twoinstitutional experience. J Thorac Dis 2017;9:151-8.

10. Fox W, Scadding JG. Medical Research Council comparative trial of surgery and radiotherapy for primary treatment of small-celled or oat-celled carcinoma of bronchus. Ten-year follow-up. Lancet 1973;2:63-5.

11. Mountain CF. Clinical biology of small cell carcinoma: relationship to surgical therapy. Semin Oncol 1978;5:272-9.

Cite this article as: $\mathrm{He} \mathrm{J,} \mathrm{Xu} \mathrm{S,} \mathrm{Pan} \mathrm{H,} \mathrm{Li} \mathrm{S,} \mathrm{He} \mathrm{J.} \mathrm{Treatments}$ for combined small cell lung cancer patients. Transl Lung Cancer Res 2020;9(5):1785-1794. doi: 10.21037/tlcr-20-437
12. Combs SE, Hancock JG, Boffa DJ, et al. Bolstering the case for lobectomy in stages I, II, and IIIA small-cell lung cancer using the National Cancer Data Base. J Thorac Oncol 2015;10:316-23.

13. Paximadis P, Beebe-Dimmer JL, George J, et al. Comparing Treatment Strategies for Stage I Small-cell lung Cancer. Clin Lung Cancer 2018;19:e559-65.

14. Yang CF, Chan DY, Speicher PJ, et al. Role of adjuvant therapy in a population-based cohort of patients with early-stage small-cell lung cancer. J Clin Oncol 2016;34:1057-64.

15. Institute NC. Surveillence, Epidemiology, and End Results. Available online: http://seer.cancer.gov/.

16. Men Y, Hui Z, Liang J, et al. Further understanding of an uncommon disease of combined small cell lung cancer: clinical features and prognostic factors of 114 cases. Chin J Cancer Res 2016;28:486-94.

17. Takei H, Kondo H, Miyaoka E, et al. Surgery for small cell lung cancer: a retrospective analysis of 243 patients from Japanese Lung Cancer Registry in 2004. J Thorac Oncol 2014;9:1140-5.

18. Bertolaccini L, Pardolesi A, Forti Parri SN, et al. Surgical approaches in patients with oligometastatic non-small cell lung cancer. J Thorac Dis 2018;10:498-502.

19. Ren Y, Dai C, Shen J, et al. The prognosis after contraindicated surgery of NSCLC patients with malignant pleural effusion (M1a) may be better than expected. Oncotarget 2016;7:26856-65.

20. Oser MG, Niederst MJ, Sequist LV, et al. Transformation from non-small-cell lung cancer to small-cell lung cancer: molecular drivers and cells of origin. Lancet Oncol 2015;16:e165-72.

21. Hakozaki T, Kitazono M, Takamori M, et al. Combined small and squamous transformation in EGFR-mutated lung adenocarcinoma: a case report. Intern Med 2020;59:1291-4. 


\section{Supplementary}

A IA-IB CSCLC Cancer-specific Survival

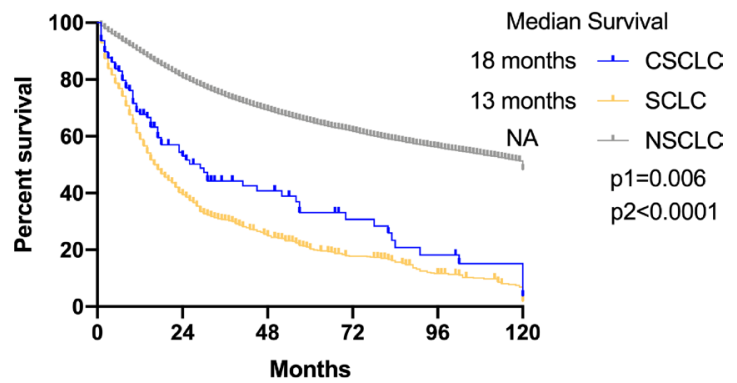

B

IIA-IIIA CSCLC Cancer-specific Survival

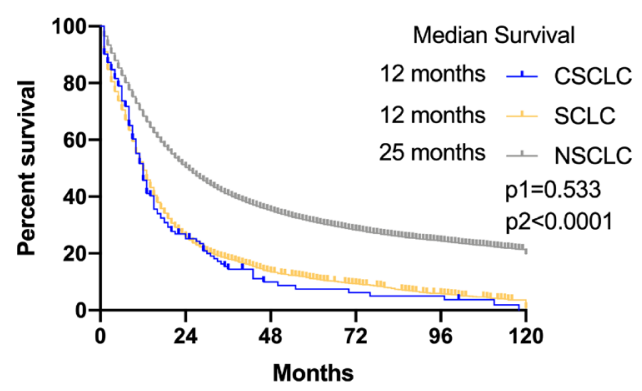

C IIIB-IV CSCLC Cancer-specific Survival

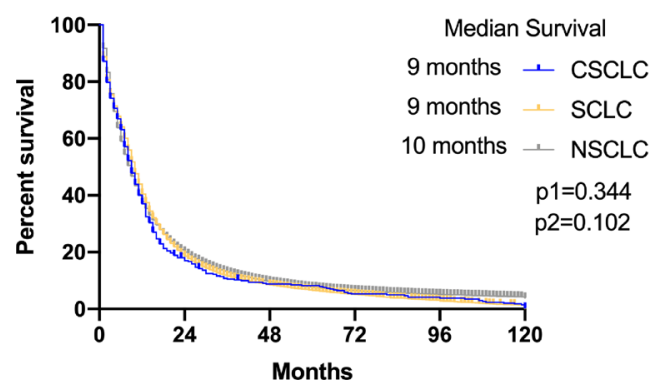

Figure S1 The survival outcomes of CSCLC, other SCLC and NSCLC patients. (A) The cancer-specific survival of stage IA-IB patients; (B) the cancer-specific survival of stage IIA-IIIA patients; (C) the cancer-specific survival of stage IIIB-IV patients. CSCLC, combined small cell lung cancer; SCLC, small cell lung cancer; NSCLC, non-small cell lung cancer. 
IA-IB CSCLC Overall Survival

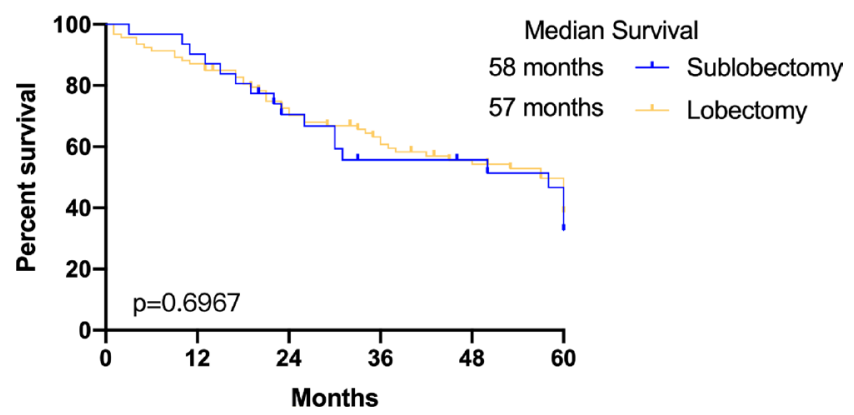

Figure S2 The overall survival of stage IA-IB CSCLC patients received sublobectomy and lobectomy. CSCLC, combined small cell lung cancer. 